\title{
Integration of Multispectral and C-Band SAR Data for Crop Classification
}

\author{
L. Iannini ${ }^{\mathrm{a}}$, R.A, Molijn ${ }^{\mathrm{a}}$, R.F. Hanssen ${ }^{\mathrm{a}}$ \\ ${ }^{a}$ Geoscience and Remote Sensing Department, Delft University of Technology, Delft, NL
}

\begin{abstract}
The paper debates the impact of sensor configuration diversity on the crop classification performance. More specifically, the analysis accounts for multi-temporal and polarimetric C-Band SAR information used individually and in synergy with Multispectral imagery. The dataset used for the investigation comprises several multi-angle Radarsat-2 (RS2) fullpol acquisitions and RapidEye (RE) images both at fine resolution collected over the Indian Head (Canada) agricultural site area and spanning the summer crop growth cycle from May to September. A quasi-Maximum Likelihood (ML) classification approach applied at per-field level has been adopted to integrate the different data sources. The analysis provided evidence on the overall accuracy enhancement with respect to the individual sensor performances, with $4 \%-8 \%$ increase over a single RE image, a $40 \%-10 \%$ increase over a single 1-pol/full-pol image and $15 \%-0 \%$ increase over multitemporal 1-pol/full-pol RS2 series respectively. A more detailed crop analysis revealed that in particular canola and the cereals benefit from the integration, whereas lentil and flax can experience similar or worse performance when compared to the RE-based classification. Comments and suggestions for further development are presented.
\end{abstract}

Keywords: Crop classification, sensor integration, maximum likelihood, SAR polarimetry.

\section{INTRODUCTION}

Reliable large-scale mapping of the crop type and crop health has a key role in economical, political ad environmental (near)real-time strategies. Remote sensing systems provide to scientists and stakeholders the unique capability of observing the land on a constant time basis at broad scales. Different spaceborne solutions have been investigated in this respect. It is common knowledge that the diversity introduced by multi-temporal observations and multiple sensor configurations significantly benefits crop characterization, since different crops respond with different temporal and spectral signatures. Data availability should be therefore considered the central issue in agricultural land classification, which indeed represents the topic of this paper. Several works have been committed to providing more insight either into SAR-based [1-3] or into optical-based [4,5] land-cover classification, trying to exploit the multi-temporal and multiband characteristics of the datasets at disposal. Further efforts have also been spent in overcoming the flaws of the individual sensors in crop identification by means of their assimilation. The availability of spaceborne SAR information is in fact currently limited by the scarce number of satellites available and by the actual sensor technology in terms of swath width/resolution performance, which determines a trade-off between revisit time and quality (resolution and polarimetric information) of the scene, while optical imagery, especially in tropical and subtropical regions, is critical due to cloud cover. Whereas single polarization SAR imaging, even in a multi-temporal context, does not provide enough reliability for operational use, its synergy with optical sensors yields significant improvements, as was for instance reported for the ERS tandem with Landsat TM [6-8], or with SPOT [9]. In the latter work, an airborne polarimetric SAR was as well tested. The benefits of polarimetry with respect of single channel SAR were indeed evident, as also proven in [3]. Only a few studies, however, has been so far committed to assess the impact of temporal/polarization and sensor diversity in land-cover classification as was done e.g. in $[9,10]$.

The present work aims therefore to provide a meaningful analysis on the accuracy achieved by polarimetric SAR and multispectral imaging indicators for different dataset combinations. The analysis is carried out on the AgriSAR 2009 dataset, presented in Section 2, which comprises multi-angle C-Band Radarsat-2 Fine Quad-Pol images, RapidEye acquisitions, and extensive ground-truth used for training and validation in the Indian Head (Canada) test site. The SAR image series has been collected during the most significant part of the crop season for a wide range of ascending and descending modes. Crop classification is performed per-field by means of a convenient quasi-ML approach. The approach, discussed in Section 3, has been applied to different sensor and acquisition setting scenarios. The objective of the analysis, presented in Section 4, is not that of performing an exhaustive exploration of the best time window/revisit

Remote Sensing for Agriculture, Ecosystems, and Hydrology XV, edited by Christopher M. U. Neale, Antonino Maltese, Proc. of SPIE Vol. 8887, 88871D · @ 2013 SPIE · CCC code: 0277-786X/13/\$18 · doi: 10.1117/12.2029330 
interval settings, but rather to focus on a relevant set of case studies that allow to effectively assess the contribute of intra- and inter-sensor diversity to crop monitoring. Some considerations on the general applicability of the analysis results to the existing and future system configurations have been provided alongside and in the conclusions.

Table I. Outline of the available SAR and MultiSpectral acquisitions employed in the analysis

\begin{tabular}{|l|l|l|}
\hline \multicolumn{1}{|c|}{ Satellite } & \multicolumn{1}{|c|}{ Beam mode } & Acquisition period (Nr of acquisitions) \\
\hline \multirow{5}{*}{ Radarsat-2 } & DSC-FQ06 & 23 April-14 September (5) \\
\cline { 2 - 3 } & DSC-FQ10 & 6 April-21 September (7) \\
\cline { 2 - 3 } & DSC-FQ19 & 20 April-11 September (6) \\
\cline { 2 - 3 } & ASC-FQ19 & 16 April-7 September (6) \\
\cline { 2 - 3 } & ASC-FQ15 & 20 April-14 September (5) \\
\cline { 2 - 3 } & ASC-FQ02 & 21 May-5 September (7) \\
\hline \multirow{2}{*}{ RapidEye } & $\begin{array}{l}5 \text { Bands } \\
\text { (Blue, Green, Red, RedEdge, NIR) }\end{array}$ \\
\hline
\end{tabular}

\section{DATA ANALYSIS}

For the analysis presented in this paper, AgriSAR 2009 data is used. The radar part of the AgriSAR 2009 data was originally acquired by Radarsat-2 for ESA in light of the upcoming ESA Sentinel-1 mission. The data package consists of Radarsat-2 quad-polarimetric data, RapidEye optical data and ground observations of test sites in Indian Head in Canada, Flevoland in the Netherlands and Barrax in Spain over the 2009 growing season.

For our research, only the data of the test site in Indian Head were used. Here, about $80 \%$ of the farmed land is zero-till with single-pass seeding and fertilization. It consists of 609 fields with 16 types of crops, which, during the acquisition time of about five months, generally experience phenological stages from bare soil to maturation. It can be stated that for the agricultural fields analyzed in this research, the bare soil and tillering stages occurs from the beginning of the studied time span (i.e. begin June), the maximum leaf area index and stem extension stage occurs at the middle of the time span (i.e. mid to end July) and the maturity/ripening stage at the end of the time span (i.e. towards September). The field areas range from 0.3 ha to 320 ha with an average field area of 73 ha.

Table I shows the number of satellite image acquisitions that were used for the analysis, with in total 36 Radarsat-2 Fine Quad-Pol Beam acquisitions and seven RapidEye images. Radarsat-2 data originally have a resolution of $5.4 \mathrm{~m}$ in slant range and $8.0 \mathrm{~m}$ in azimuth direction, whereas the RapidEye images have a resolution of $6.5 \mathrm{~m}$ in five spectral bands (RGB, Red-Edge and NIR). All the acquisitions have undergone the pre-processing steps illustrated in Figure 1, and here listed:

- Radiometric calibration of both the SAR and optical images, this latter were corrected to the Top of Atmosphere (TOA) reflectance. Additional filtering and pre-processing operations might be applied, such as the Sentinel-1 data simulation applied to the RS2 data by the AgriSAR team to adapt the level of noise and ENL (Equivalent number of Looks) to the Sentinel-1 reference values.

- Geocoding of both datasets into the same geographic coordinate frame. The data were projected into a $10 \times 10 \mathrm{~m}$ grid by using a SRTM DEM.

- Field segmentation and masking of the areas not affected by cloud cover.

Only those fields covered by all the SAR and all RapidEye images were included in the analysis. For this research, of the 16 types of crops available a selection was made and various (similar) types of crops were aggregated before training and classification. We omitted the crop types that are represented by a small number of fields and were not suitable for aggregation (resulting in the omission of alfalfa). As for barley, oat, spring wheat, durum wheat, rye and summer fallow, 
these were aggregated into the cereals class. Canary seed, mixed hay, mixed pasture, chemical fallow and grass were aggregated into the grasses class. Canola, field pea, lentil and flax were all treated as individual classes.

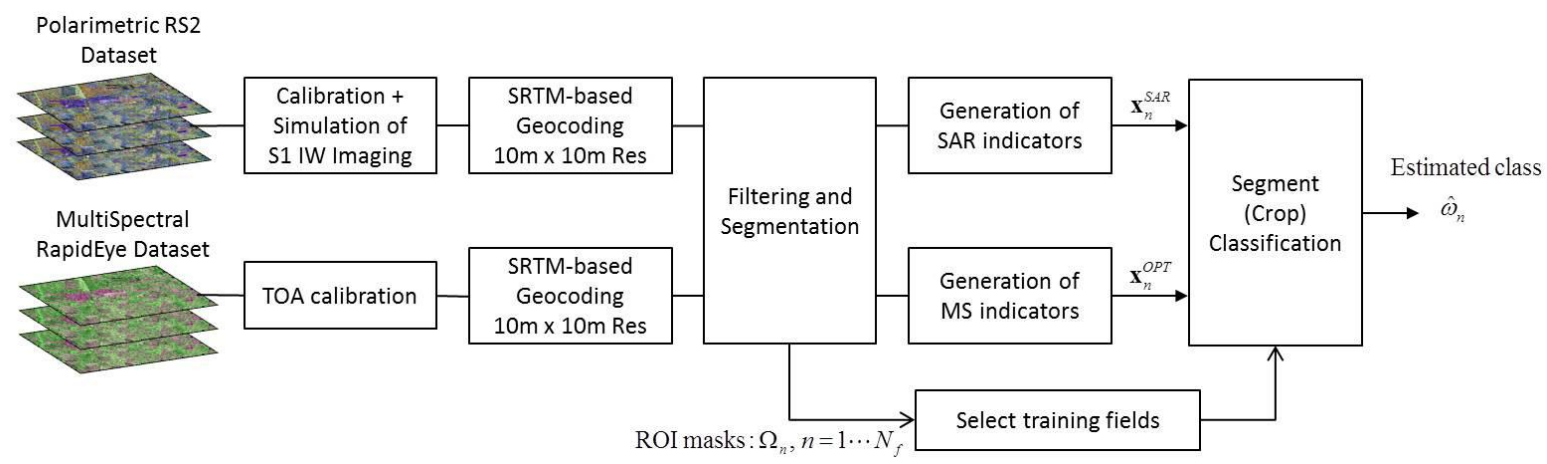

Figure 1. Schematic representation of the classification procedure adopted.

\section{CLASSIFICATION APPROACH}

For this research, the field classification is based on the Maximum Likelihood (ML) supervised method, which is a convenient and widely used approach when reliable information on the probability density function (pdf) of the data is available. On the other hand, as a parametric classifier, it is sensitive to inaccuracies in the probability model of the data and to the errors in the parameters estimates, i.e. in the quality and quantity of the samples during the training procedure. The classification is applied to the area segments (fields) resulting from the processing chain of Figure 1. A per-field approach instead of a pixel-based classification has been adopted, as suggested by [4,5].A fixed conservative ratio of $1 / 2$ between training and validation set lengths has been chosen. The problem hence addressed is that of modeling the likelihood function for the segment feature vector:

$$
\mathbf{x}=\left[\begin{array}{c}
\mathbf{x}_{1}^{S A R} \\
\vdots \\
\mathbf{x}_{N_{S A R}}^{S A R} \\
\mathbf{x}_{1}^{\text {OPT }} \\
\vdots \\
\mathbf{x}_{N_{\text {OPP }}}^{\text {OPT }}
\end{array}\right]
$$

which comprises both polarimetric SAR observations $\mathbf{x}^{\text {SAR }}$ and optical sensor observations $\mathbf{x}^{\text {OPT }}$, within a stack of $N_{S A R}$ and $N_{O P T}$ images. With respect to SAR, the target represented by the crop field must be regarded as a distributed scatterer. This means that the signal observed is governed by the coherent combination of multiple mechanisms within each resolution cell, which is referred to as speckle. Rather than dealing with the single cell content $\mathbf{s}=\left[S_{H H}, S_{H V}, S_{V V}\right]^{T}$, it is more convenient to work on its second order information, i.e. the polarimetric covariance matrix $\mathbf{C}$.

$$
\mathbf{C}=\frac{1}{K} \sum_{i=1}^{K} \mathbf{s} \cdot \mathbf{s}^{H}=\left[\begin{array}{ccc}
\left\langle\left. S_{H H}\right|^{2}\right\rangle & \left\langle S_{H H} S_{H V}^{*}\right\rangle & \left\langle S_{H H} S_{V V}^{*}\right\rangle \\
\left\langle S_{H V} S_{H H}^{*}\right\rangle & \left\langle\left. S_{H V}\right|^{2}\right\rangle & \left\langle S_{H V} S_{V V}^{*}\right\rangle \\
\left\langle S_{V V} S_{H H}^{*}\right\rangle & \left\langle S_{V V} S_{H V}^{*}\right\rangle & \left\langle\left|S_{V V}\right|^{2}\right\rangle
\end{array}\right]
$$

where ${ }^{H}$ stands for the Hermitian transpose operator and $<$. $>$ refers to the spatial average over the $K$ cells of the field area. The covariance matrix behaves in accordance with the complex Wishart distribution $C \mathcal{W}(\mathbf{C}, n)[11]$, under the 
assumption that it is a result of fully developed speckle and scattering homogeneity within the field segment area of $n$ independent looks. The analytic expression of the Wishart pdf is well-known, however its sequent merging with the optical data statistics is not necessarily straightforward. Besides, it should be noted that a Wishart-based likelihood does not effectively account for intra-crop diversity, which can be caused by slight misalignments in growth stages or different soil and weather conditions. In order to robustly tackle such issues, the approach proposed in [12] is adopted for this paper. It exploits the fact that when the number of looks $n$ is high enough, the gamma-distributed polarimetric intensities can be closely approximated by a log-normal behavior [13]. The polarimetric information related to the crosspolarization covariance terms (out of the diagonal) is retained by introducing the intensity of additional polarization modes. Such intensities are easily determined by a reversible linear conversion from the real components of $\mathbf{C}$. It will be then supposed that reflection symmetry assumption, i.e. $\left\langle S_{H V} S_{H H}^{*}\right\rangle=0$ and $\left\langle S_{H V} S_{V V}^{*}\right\rangle=0$, is applicable to crop fields.

This is true in general on average, though it was reported that few crops at some specific growth stage, wind and tillage conditions present a small asymmetric component, leading to the consideration that the observations might be retained in such cases only for applications such as phenology tracking. In our analysis only the 5 real elements:

$$
\boldsymbol{\sigma}=\left[\begin{array}{c}
\sigma_{H H}^{0} \\
\sigma_{H V}^{0} \\
\sigma_{V V}^{0} \\
\sigma_{+-45}^{0} \\
\sigma_{+45 l}^{0}+\sigma_{-45 r}^{0}
\end{array}\right]=\mathbf{B} \cdot\left[\begin{array}{c}
\left\langle\left|S_{H H}\right|^{2}\right\rangle \\
\left\langle\left|S_{H V}\right|^{2}\right\rangle \\
\left\langle\left|S_{V V}\right|^{2}\right\rangle \\
R\left\{\left\langle S_{H H} S_{V V}^{*}\right\rangle\right\} \\
I\left\{\left\langle S_{H H} S_{V V}^{*}\right\rangle\right\}
\end{array}\right]
$$

will be considered, where $\mathbf{B}$ is the conversion matrix and where the following polarization bases are introduced: ()$+$ and - $45^{\circ}$ rotated linear polarization, (l) left circular and (r) right circular. Such approach would allow operating on the polarimetric information in a more comfortable context, where the pdf of a single acquisition can be written as:

$$
p_{\mathbf{x}}\left(\mathbf{x}^{S A R}\right)=\frac{1}{(2 \pi)^{P / 2}|\boldsymbol{\Gamma}|^{1 / 2}} \exp \left(-\frac{1}{2}\left(\mathbf{x}^{S A R}-\boldsymbol{\eta}^{S A R}\right)^{T} \boldsymbol{\Gamma}^{-1}\left(\mathbf{x}^{S A R}-\boldsymbol{\eta}^{S A R}\right)\right)
$$

with $\mathbf{x}^{S A R}=\left.\boldsymbol{\sigma}\right|_{d B}$, and $\boldsymbol{\eta}^{S A R}, \boldsymbol{\Gamma}$ standing for the mean value and covariance matrix of $\mathbf{x}^{S A R}$.

With regard to the multiband optical data, the feature vector is composed by the 5 optical bands of the RapidEye sensor (i.e. RGB, Red-Edge and NIR) and averaged over the field segment. It has to be remarked that no phenomenological model can be easily associated with the received signal fluctuations. These have to be considered as variations of the local reflectance in the different bands, also since sensor noise would not make a significant contribution to these fluctuations. Since a theoretical justification upon the validity of a chosen model appears unfeasible, a reasonable solution based on empirical criteria could be the best way to proceed, as suggested in [14]. It was then assessed that the log-normal distribution provides a good fit to the plain optical band data. The likelihood assigned to each feature vector

$$
\mathbf{x}^{O P T}=\left[\begin{array}{lll}
\ln \mathrm{x}_{1} & \cdots & \ln \mathrm{x}_{\mathrm{N}_{\text {bands }}}
\end{array}\right]^{T}
$$

with $\mathrm{x}_{\mathrm{i}}$ referring to field segment observation by the $i$-th optical band, takes as a result the same expression of the pdf in (4). The overall likelihood $L$ is then assembled by considering the acquisitions independent, both within the same sensor and among the different sensors, yielding consequently the quasi-ML approximation:

$$
\ln L\left(\mathbf{x}, \omega_{i}\right)=\sum_{n=1}^{N_{S A R}} \ln p_{\mathbf{x}}\left(\mathbf{x}_{n}^{S A R} \mid\left\{\boldsymbol{\eta}_{n}^{S A R}(i), \boldsymbol{\Gamma}_{n}^{S A R}(i)\right\}\right)+\sum_{n=1}^{N_{O P T}} \ln p_{\mathbf{x}}\left(\mathbf{x}_{n}^{O P T} \mid\left\{\boldsymbol{\eta}_{n}^{O P T}(i), \boldsymbol{\Gamma}_{n}^{O P T}(i)\right\}\right)
$$

Where $\ln L$ is the $\log$-likelihood function and where $\omega_{i}$ represents the $i$-th class, whose signature $\left\{\boldsymbol{\eta}_{n}^{S A R}(i), \boldsymbol{\Gamma}_{n}^{S A R}(i), \boldsymbol{\eta}_{n}^{S A R}(i), \boldsymbol{\Gamma}_{n}^{O P T}(i)\right\}$ consists in a reference temporal behavior of the mean and covariance matrix parameters. The classifier then trivially assigns to the sample $\mathbf{x}$ the class $i$ with maximum log-likelihood. The 
independency assumption is fundamental in order to restrain the training field subset to a reasonable size. This means that when the full covariance information in each $\Gamma_{n}^{S A R}, \boldsymbol{\Gamma}_{n}^{O P T}$ is used, the application of (6) requires the number of training fields to be larger than both the number of SAR polarizations, and the number of optical bands.

\section{PERFORMANCE ANALYSIS}

The AgriSAR dataset delivers the potential for assessing a very large number of interesting case studies. However, the aim of the analysis is not to cover every possible sensor diversity but rather to focus on the ones that deliver the most relevant insight on the sensor integration procedure. The reader should as well note that the rationale supporting such investigation concerns the actual lack of timeliness in high resolution optical imaging due to cloud cover, in front of the reliability of SAR sensors, which however offer much poorer performance when polarimetric acquisitions are not involved. For every sensor configuration tested, the associated results shown have been retrieved out of $N_{r}=100$ algorithm runs, each run performing a random selection of the training set, in accordance to Figure 1 scheme. The overall accuracy and the producer/consumer accuracy for each class are used as performance criterions, defined respectively as $\sum_{i} \sum_{r} n_{r}\left(i \mid \omega_{i}\right) \sum_{r} n_{r}\left(i \mid \omega_{i}\right) \quad \sum_{r} n_{r}\left(i \mid \omega_{i}\right)$, where $n_{r}\left(i \mid \omega_{j}\right)$ is the number of samples assigned to class $i$ and belonging to class $j$

$$
\frac{i, r}{N_{r} \cdot \sum_{i} N_{i}}, \frac{r}{N_{r} N_{i}}, \frac{r}{\sum_{j} \sum_{r} n_{r}\left(i \mid \omega_{j}\right)}
$$

and $N_{i}$ is the total number of samples in class $i$.

The analysis will first focus on the performance attained by combining a single RS2 acquisition with a single RE image. The RE image is fixed throughout the simulations whereas the temporal diversity is explored with respect to the SAR sensor. The classification accuracy individually achieved by each optical acquisition is reported in Figure 2 left panel. It is evident that the images collected during the development stages of the crops outperform, with overall accuracy $>91 \%$, the ones framing crop development stages $(86 \%)$ or (pre)germination stages (67\%). The last optical acquisition $(05 / 09 / 2009)$ has been chosen for the combined sensor analysis, thus simulating the worst-case scenario, i.e. severe cloud cover conditions throughout large part of the crop growth. The worst and best performance SAR polarimetric modes, i.e. the single-pol mode (HH) and the full-pol mode described by (5) respectively, are shown in Figure 2 right panel. The performance is evaluated for RS2 alone and for the RS2+RE combination on all the available images regardless of the beam mode.

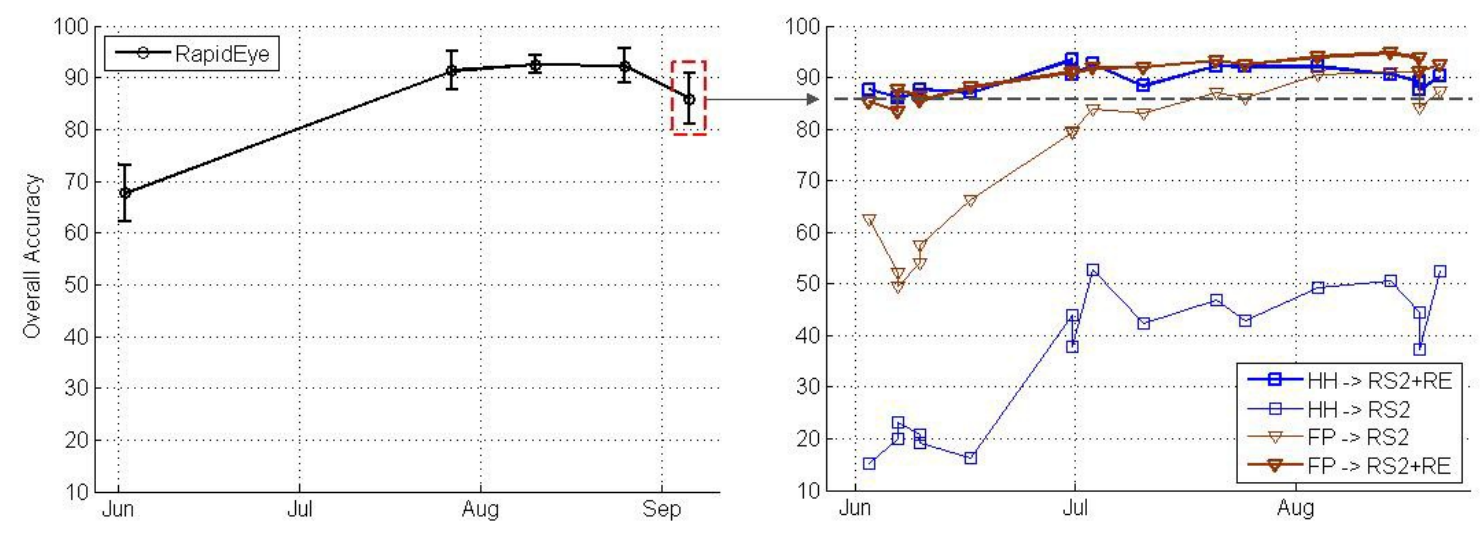

Figure 2. (left) Overall accuracy achieved by each individual RapidEye acquisition. The standard deviation, computed over 100 simulations is reported as well through the use of error bars. (right) RS-2 performance reported as a function of the acquisition time, when processed independently and when integrated with the RE image marked by the red box in the left panel. 

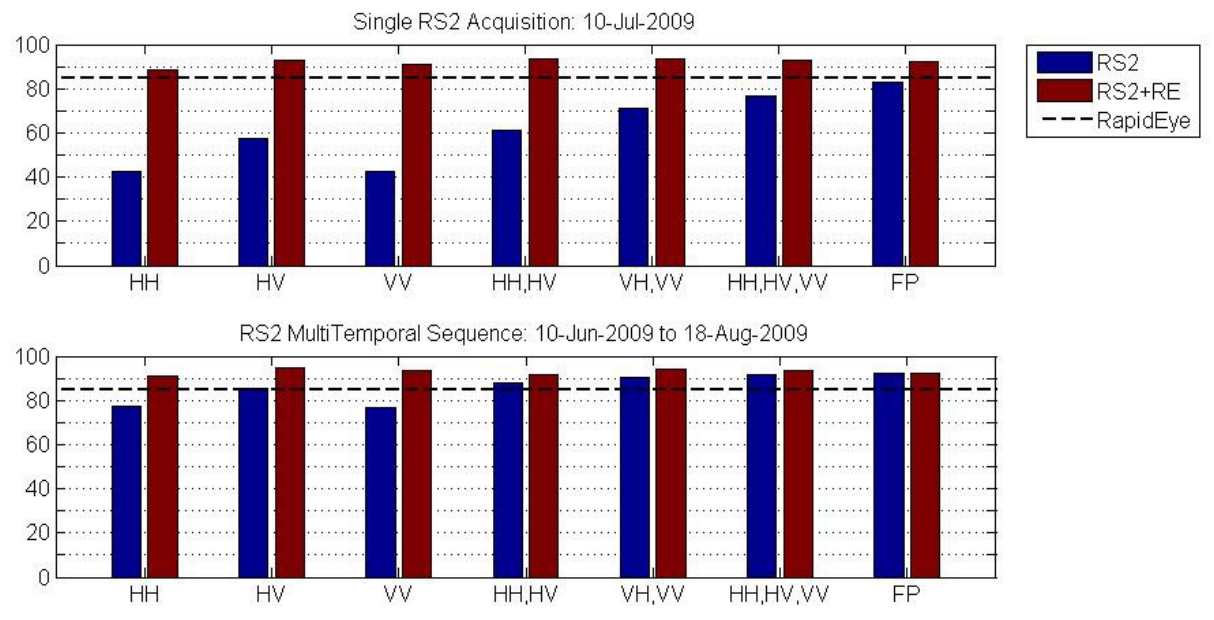

Figure 3. Overall accuracy achieved by two different SAR configurations, a single July acquisition (top) and a series of 11 images with 7-days revisit (bottom), when processed with and without and integrated with the 05-Sep-2009 RapidEye acquisition. Different polarimetric modes are assessed: single, dual and quad-pol, this latter including the simple $\mathrm{HH}, \mathrm{HV}, \mathrm{VV}$ combination and the full polarimetric information, FP, where the 5 intensities in (3) are accounted.

Single-image (SI) joint classification reaches accuracy of approximately $92 \%$ for single-pol and $94 \%$ for full-pol mode, marking a 6-8\% performance enhancement over the RapidEye results. The benefits can be noticed starting from July, whereas neither significant improvement nor degradation is visible when the information brought by the SAR is poor, as expected in the first part of the sequence. The results so far presented (single SAR image) represent the lower bound on SAR classification performance. Classification based on multi-image (MI) SAR information will be hence introduced. For the sake of a sharp and focused discussion, we chose to base the forthcoming analysis on the best case configuration: the data-takes collected within the 2-month time frame ranging from 10 June to 18 August were have been selected, i.e. a subset of 11 images with average revisit interval of 7 days, close indeed to the Sentinel-1 8-days cycle. Classification performance computed on such image set for the polarimetric configurations is compared in Figure 3 with the performance achieved by the same modes on a single mid-July acquisition. The difference in accuracy between the several polarimetric modes is evident when SAR-only classification is applied, with the HV registering the best results in 1-pol configuration (54\% in SI vs $85 \%$ in MI ), VH-VV scoring the best accuracy (71\% in SI vs $91 \%$ in MI) in dual-pol in agreement with other reference works [3], and the set of polarimetric powers in (5), recalled as FP, attaining the best results $(83 \%$ in SI vs $92 \%$ in MI) with quad-pol. Differently, it can be noticed that the overall accuracy is leveled as a result of the sensor integration, with almost every polarimetric mode and image setting registering beyond $90 \%$. This naturally leads to the consideration that in crop classification the information complementarity with respect to the optical bands is effectively provided by single-pol and dual-pol SAR data.

Despite the differences can be hardly considered as significant in absolute terms, it is nonetheless interesting to notice that the FP mode is not leading in terms of performance, for instance with respect to both the HV and VH-VV modes. A more exploded analysis on the single class accuracies has been therefore conducted for these three modes. The outcome, shown in Figure 4, highlights the fact that both omission and commission error are reduced by the sensor integration in each class when compared to the SAR-only classification. The same analysis does not apply to the RapidEye case, where only the canola and cereal classes are clearly benefiting from the RS2 data. It might as well be noticed that in the FP mode, the omission error of lentil and flax is significantly increased with respect to both RapidEye-only and single-/dualpol modes. The additional FP information is therefore introducing ambiguity between classes in such case. It shall be nonetheless noticed that the number of samples used for training (which is the same of that used for classification, since a $1 / 2$ ratio is adopted) is significantly low, 8 for lentil and 6 for flax, thus generating large uncertainty in the reference mean and covariance signature estimates. The last considerations open indeed an interesting point, marking the need of a better information merging between SAR and optical when the balance, in terms of number of features, is significally different among the two sensors, such as in the fusion of multi-temporal polarimetric SAR data and a single optical image. While retaining a parametric approach, a more efficient data weighting (time- and sensor-adaptive) based on further scene-based experimental or a-priori information might be adviced. Additionally, different performance 
indicators such as the likelihood ratio between the first and second most likely classes might be included into a more refined thresholding approach. Further research on such issues seems promising and is currently under investigations.

\section{CONCLUSIONS}

The paper promotes a debate on the supposition that multi-source data can deliver within land-cover, and more specifically crop type, mapping application. The analysis is aimed at shedding light on the class discrimination potential of each sensor, when evaluated individually and in a joint scenario. A per-field quasi-ML approach was adopted to assimilate the segment features extracted by the different image sources. Individual sensor performance showed a very high accuracy, $>90 \%$, achieved by RapidEye (RE) imaging, that could be matched by SAR only in the case of multitemporal full-polarimetric series. With regard the sensor integration, we decided to design the simulation scenario by accounting only for the single RE acquisition collected in September at the end of the maturation cycle, thus picturing an unfortunate scenario where cloud cover makes all the best images inaccessible. The sensor integration could achieve a $4 \%-8 \%$ accuracy increase over the RE-only acquisition, and a 40\%-0\% increase range over the RS2 modes with $40 \%$ measured for $\mathrm{HH} 1$-pol, and $0 \%$, i.e. no improvement, registered for the full-pol 2-months series. It was furthermore observed that most of the benefits from sensor integration concern canola and the cereals, whereas the other crop types, e.g. lentil and flax, can experience similar or worse performance when compared to the RE-based classification. In light of such results, a more refined classification approach, where the multi-source data is weighted as a result of additional a-priori information (from models or empirical signature observation), might be advised. A deeper study on the polarimetric and multispectral crop signature diversity and a more extended error analysis on both Indian Head and also the Flevoland (NL) scenario will be presented at the conference in support of these final considerations.

\section{ACKNOWLEDGMENTS}

The data used for the analysis was originally acquired and preprocessed within the frame of the AgriSAR 2009 ESA campaign. Access to such data from ESA is therefore gratefully acknowledged.

\section{REFERENCES}

[1] F. Del Frate, G. Schiavon, D. Solimini, M. Borgeaud, D. H. Hoekman, and M. A. M. Vissers, "Crop classification using multiconfiguration C-band SAR data," IEEE Trans. Geosci. Remote Sens. 41(7), 1611-1619 (2003).

[2] H. McNairn and B. Brisco, "The application of C-band polarimetric SAR for agriculture: a review," Can. J. Remote Sens. 30(3), 525-542 (2004).

[3] H. Skriver, "Crop Classification by Multitemporal C- and L-Band Single- and Dual-Polarization and Fully Polarimetric SAR,” IEEE Trans. Geosci. Remote Sens. 50(6), 2138-2149 (2012).

[4] M. Turker and M. Arikan, "Sequential masking classification of multi temporal Landsat7 ETM+ images for field based crop mapping in Karacabey, Turkey," Int. J. Remote Sens. 26(17), 3813-3830 (2005).

[5] A. J. W. De Wit and J. G. P. W. Clevers, "Efficiency and accuracy of per-field classification for operational crop mapping," Int. J. Remote Sens. 25(20), 4091-4112 (2004).

[6] B. Brisco and R. J. Brown, "Multidate SAR/TM synergism for crop classification in western Canada," Photogramm. Eng. Remote Sens. 61(8), 1009-1014.

[7] D. B. Michelson, B. M. Liljeberg, and P. Pilesjö, "Comparison of algorithms for classifying Swedish landcover using Landsat TM and ERS-1 SAR data," Remote Sens. Environ. 71(1), 1-15 (2000).

[8] Y. Ban, "Synergy of multitemporal ERS-1 SAR and Landsat TM data for classification of agricultural crops," Can. J. Remote Sens. 29(4), 518-526 (2003).

[9] I. Sandholt, "The combination of polarimetric SAR with satellite SAR and optical data for classification of agricultural land," Geogr. Tidsskr.-Dan. J. Geogr. 101(1), 21-32 (2001). 
[10] M. J. Hill, C. J. Ticehurst, J.-S. Lee, M. R. Grunes, G. E. Donald, and D. Henry, "Integration of optical and radar classifications for mapping pasture type in Western Australia," IEEE Trans. Geosci. Remote Sens. 43(7), 1665$1681(2005)$.

[11] J.-S. Lee, K. W. Hoppel, S. A. Mango, and A. R. Miller, "Intensity and phase statistics of multilook polarimetric and interferometric SAR imagery," IEEE Trans. Geosci. Remote Sens. 32(5), 1017-1028 (1994).

[12] D. H. Hoekman and M. A. M. Vissers, "A new polarimetric classification approach evaluated for agricultural crops,” IEEE Trans. Geosci. Remote Sens. 41(12), 2881-2889 (2003).

[13] D. H. Hoekman, "Speckle ensemble statistics of logarithmically scaled data [radar]," IEEE Trans. Geosci. Remote Sens. 29(1), 180-182 (1991).

[14] P. Lombardo, C. J. Oliver, T. M. Pellizzeri, and M. Meloni, "A new maximum-likelihood joint segmentation technique for multitemporal SAR and multiband optical images," IEEE Trans. Geosci. Remote Sens. 41(11), 2500-2518 (2003).
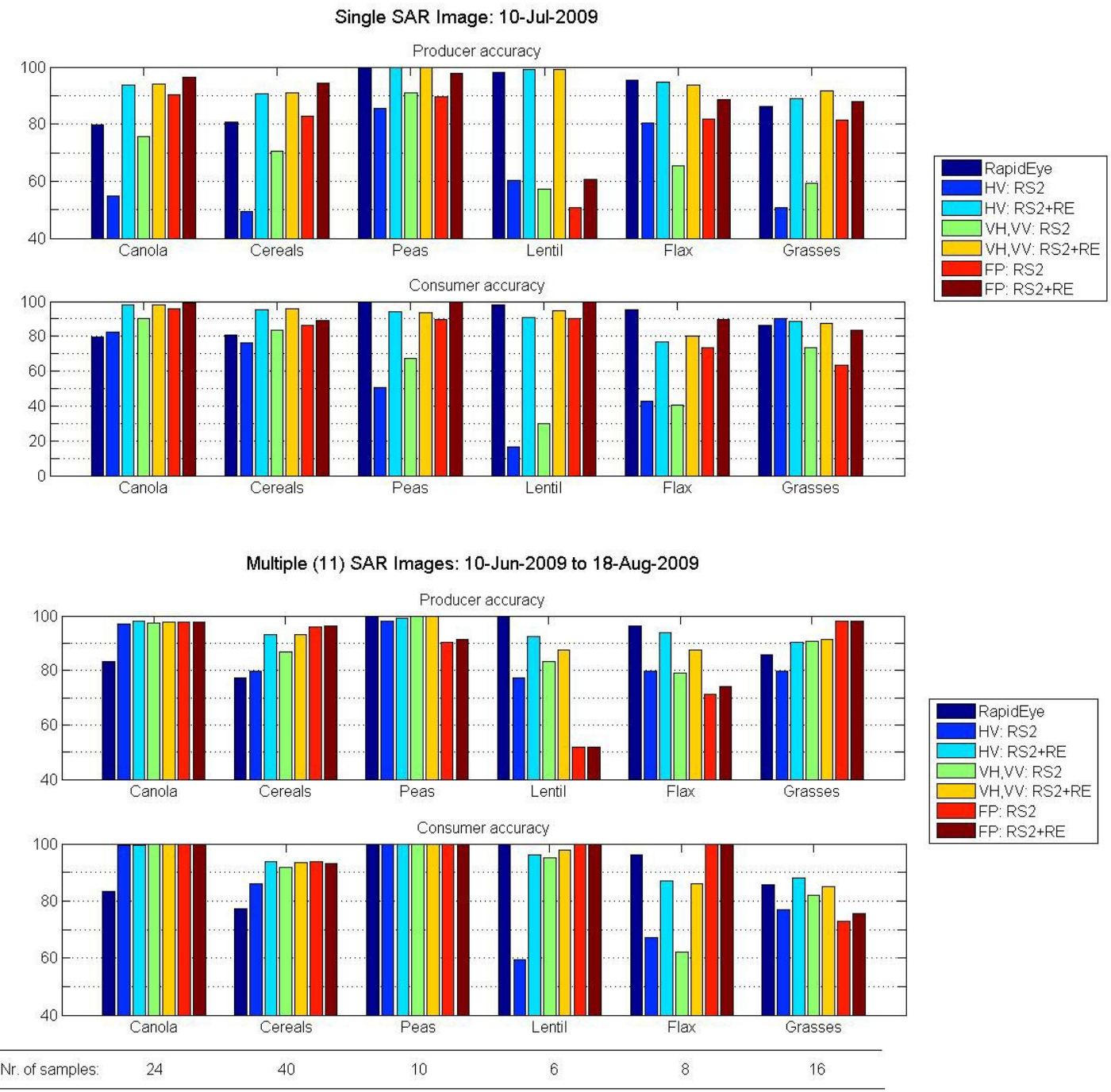

Figure 4. Producer and consumer accuracy attained by 3 representative polarimetric combinations in the single-image mode (top panels) and multi-image mode (bottom panels) for each crop type. The number of fields available for validation is reported in the lower portion of the figure. 